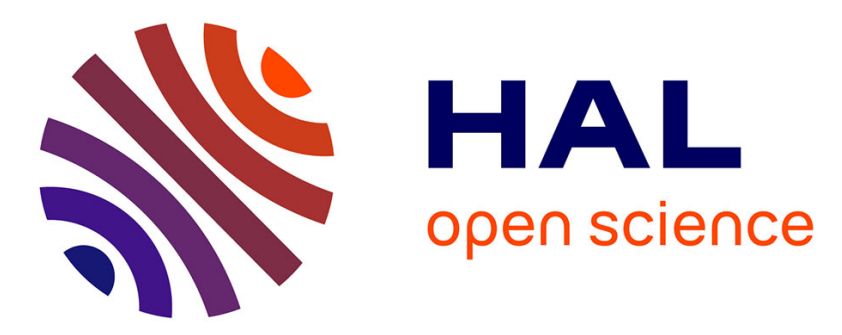

\title{
Égalité professionnelle et genre en contexte : l'exemple des chef.fes d'établissement du secondaire
}

\author{
G. Combaz, C. Morin-Messabel
}

\section{To cite this version:}

G. Combaz, C. Morin-Messabel. Égalité professionnelle et genre en contexte : l'exemple des chef.fes d'établissement du secondaire. Psychologie du travail et des organisations, 2021, 27 (2), pp.89-104. 10.1016/j.pto.2021.02.001 . hal-03348712

\section{HAL Id: hal-03348712 \\ https://hal.univ-lyon2.fr/hal-03348712}

Submitted on 20 Sep 2021

HAL is a multi-disciplinary open access archive for the deposit and dissemination of scientific research documents, whether they are published or not. The documents may come from teaching and research institutions in France or abroad, or from public or private research centers.
L'archive ouverte pluridisciplinaire HAL, est destinée au dépôt et à la diffusion de documents scientifiques de niveau recherche, publiés ou non, émanant des établissements d'enseignement et de recherche français ou étrangers, des laboratoires publics ou privés. 


\title{
Égalité professionnelle et genre en contexte : l'exemple des chef.fes d'établissement du secondaire
}

\section{Professional equality and gender in context: The example of secondary school head teachers}

\author{
G. Combaz ${ }^{\mathrm{a}}$, C. Morin-Messabel ${ }^{\mathrm{b}, *}$
}

${ }^{a}$ Laboratoire éducation, cultures, politiques (EA 4571), institut des sciences et des pratiques d'éducation et de formation (ISPEF), université Lumière Lyon-II, Lyon, France

${ }^{\mathrm{b}}$ Laboratoire GRePS (EA 4163), institut de psychologie, université Lumière Lyon-II, Lyon, France

\section{N F O A R T I C L E}

\section{Historique de l'article :}

Reçu le 21 décembre 2020

Reçu sous la forme révisée

le 21 janvier 2021

Accepté le 16 février 2021

\section{Mots clés :}

Égalité professionnelle Perception des inégalités Chef.fe d'établissement Genre

\section{R É S U M É}

Au sein de la fonction publique, le secteur de l'enseignement primaire et secondaire est très féminisé. Notre objet d'étude est en lien avec ces questions d'égalité professionnelle au sein de l'Éducation nationale (contexte fortement féminisé) et vise à explorer le discours tenu par les personnels de direction (et notamment les femmes) au niveau de la perception (ou non) d'inégalités femmes/hommes dans l'accès aux postes d'encadrement (perception subjective des inégalités). Les résultats permettent tout d'abord de montrer l'hétérogénéité des réponses des personnels (y compris des femmes) sur les questions d'égalité perçue. Comparativement, une majorité des personnes interrogées ont souligné les difficultés d'articuler les différents temps de vie (professionnel, familiaux, personnels). Elles ont également mis l'accent sur la disponibilité et l'engagement que requiert l'exercice de fonction de chef d'établissement. Et, dans ce registre, il semble exister un relatif accord entre les femmes et les hommes qui ont répondu (même si, du point de vue quantitatif, les femmes sont plus nombreuses à évoquer ces aspects). Les champs d'application de ces recherches sont à mettre en lien avec les politiques d'égalité dans la fonction publique et les plans d'actions en faveur de l'égalité femmes/hommes.

(c) 2021 AIPTLF. Publié par Elsevier Masson SAS. Tous droits réservés.

\footnotetext{
* Auteur correspondant.

Adresses e-mail : gilles.combaz@univ-lyon2.fr (G. Combaz), Christine.morin-messabel@univ-lyon2.fr (C. Morin-Messabel).
} 
Keywords:

Professional equality

Perception of inequalities

Head teacher

Gender

\section{A B S T R A C T}

In the public service, the elementary and secondary education sector is highly feminised. Our object of study is related to these issues of professional equality within the national education system (a highly feminised context) and aims to explore the discourse held by management staff (especially women) on the perception (or not) of gender inequalities in access to managerial positions (subjective perception of inequalities). The results make it possible first of all to show the heterogeneity of the responses of staff (including women) on questions of perceived equality. Comparatively, a majority of the interviewees emphasised the difficulties in articulating different life times (professional, family, personal). They also emphasised the availability and commitment required to carry out the function of head teacher. And, in this respect, there seems to be relative agreement between the women and men who responded (although, from a quantitative point of view, more women mentioned these aspects). The fields of application of this research should be linked to equality policies in the public service and action plans for gender equality.

(c) 2021 AIPTLF. Published by Elsevier Masson SAS. All

rights reserved.

\section{Introduction}

En mars 2020, la Commission européenne a impulsé une nouvelle stratégie en faveur de l'égalité entre les hommes et les femmes pour la période 2020-2025. Il s'agit d'améliorer « l'équilibre hommesfemmes dans les professions tendant traditionnellement à être dominées par les hommes ou les femmes, de lutter contre les stéréotypes sexistes et de s'attaquer aux inégalités entre hommes et femmes en matière d'éducation et de formation ". Cette volonté européenne sur les questions d'égalité et de mixité se traduit également au niveau des personnels de l'éducation. En France, la loi du 6 août 2019 en lien avec l'accord du 30 novembre 2018 relatif à l'égalité professionnelle entre les femmes et les hommes dans la fonction publique implique la mise en place de plan d'action au niveau des ministères de l'Éducation nationale et de la Jeunesse et celui de l'Enseignement supérieur, de la Recherche et de l'Innovation en matière d'égalité. Plus précisément, les grands chantiers issus de cette loi concernent les écarts de rémunération entre les femmes et les hommes, les carrières des personnels, l'égal accès des femmes et des hommes aux corps, grades et emplois, l'articulation entre activité professionnelle et vie personnelle et familiale et enfin la prévention des discriminations, actes de violence, de harcèlement moral ou sexuel ainsi que les agissements sexistes.

Au sein de la fonction publique, le secteur de l'enseignement primaire et secondaire est très féminisé. C'est un peu moins le cas pour l'enseignement supérieur. Cette féminisation - entendue ici au sens quantitatif du terme - concerne-t-elle l'ensemble des personnels, en particulier ceux qui occupent des postes d'encadrement ? Un rapport récent (Bidar et al., 2016) montre que ce n'est pas le cas pour les postes les plus élevés (recteurs, responsables d'administrations déconcentrées, inspecteurs généraux). En revanche, la répartition par sexe pour l'encadrement intermédiaire est plus équilibrée. Entre 1998 et 2017, la part des femmes cheffes d'établissements du second degré est passée de 32,5 \% à 48,9 \% (MEN - DEPP). Nous observons la même tendance pour les inspectrices de l'enseignement primaire : elles représentent 30,7 \% de l'ensemble en 1998 et 48,9\% en 2017. Pour ces deux corps de fonctionnaires, l'équilibre est quasiment atteint. La direction d'écoles dans l'enseignement du premier degré qui s'apparente davantage en France à un encadrement de 
proximité, est également très féminisée (71,7 \% de femmes en 2005 et 74,8 \% en 2016. MEN - DEPP). Mais les probabilités d'accéder à ce type de poste sont plus élevées pour les hommes (Combaz \& Burgevin, 2015).

La question de recherche traitée ici est en lien avec ces questions d'égalité professionnelle au sein de l'Éducation nationale (contexte fortement féminisé) et vise à explorer le discours tenu par les personnels de direction (et notamment les femmes) au niveau de la perception (ou non) d'inégalités femmes/hommes dans l'accès aux postes d'encadrement. Cette recherche est exploratoire et mobilise un regard croisé de la sociologie des inégalités et de la psychologie sociale en contexte professionnel. Au-delà de ce regard croisé, notre objet est de montrer la complexité des dynamiques sociales inégalitaires perçues (ou non) par les acteurs-actrices « ce qu'ils.elles en pensent » : "Or, c'est parfois en faisant confiance aux acteurs, à ce qu'ils disent, que l'on peut dégager une meilleure compréhension des relations entre sexes. " (Tostain, 2016, p. 356). Notre objet d'étude concerne aussi la question de la prise de conscience des mécanismes formels ou informels susceptibles d'affecter les carrières professionnelles des femmes dans le discours des personnels interrogés. En effet, comme le souligne Alber (2013) dans son article, au-delà de données quantitatives, il devient nécessaire de recueillir des données qualitatives permettant d'éclairer la mise en place d'obstacles aux femmes dans l'accès aux fonctions de direction dans la fonction publique voire de mettre en lumière les phénomènes d'autocensure chez les femmes dans des responsabilités d'encadrement.

\subsection{Inégalités objectives/subjectives en sciences humaines et sociales}

Dans le champ des sciences sociales, l'étude des inégalités objectives représente une thématique traditionnellement très développée (Galland \& Lemel, 2018). En revanche, la perception subjective des inégalités et la façon dont elles peuvent être interprétées par les acteurs n'ont été que très récemment explorées par les chercheurs. En France, il faut attendre la fin des années 1970 pour qu'un premier rapport soit publié par les membres du Groupe d'étude des méthodes de l'analyse sociologique (GEMAS, 1976). Cette perspective va ensuite être développée par un certain nombre d'économistes et de sociologues qui vont s'intéresser à la perception de différents types d'inégalités : les inégalités économiques (Forsé \& Parodi, 2007), les inégalités culturelles (Coulangeaon, 2016), les rémunérations (Forsé, 2011), les revenus (Gonthier, 2011), la stratification sociale (Marcel \&Verley, 2011), les inégalités liées à l'origine ethnique (Collet \& Keucheyan, 2011), la mobilité sociale (Duru-Bellat \& Kieffer, 2006).

\subsection{Inégalités et plafond de verre}

L'accord du 30 novembre 2018 relatif à l'égalité professionnelle entre les femmes et les hommes dans la fonction publique vise à rendre effective l'égalité entre les femmes et les hommes dans les rémunérations, les parcours et responsabilités professionnelles. Ce qui n'est pas encore le cas dans les organisations y compris la fonction publique. Laufer (2005) dans son article portant sur les femmes cadres à haut potentiel, définit le " plafond de verre " comme l'ensemble des obstacles visibles et surtout invisibles qui séparent les femmes du sommet des hiérarchies professionnelles et organisationnelles. Elle souligne l'existence dans les organisations de relations de pouvoir et de processus informels, souvent inégalitaires qui déterminent l'accès à ces postes. Du point de vue psychosocial, les mécanismes caractéristiques du plafond de verre qui entrent en jeu dans ce " plafonnement " relèvent comme le résume Latour (2008) d'éléments stéréotypés : les femmes seraient perçues comme moins ambitieuses, leaders, combatives, plus sujettes à l'autocensure et se consacrant davantage à leur famille que les hommes. Laufer (2005) identifie dans son enquête auprès de femmes cadres à haut potentiel des facteurs impactant l'évolution de carrière des femmes : le profil du poste occupé, le management et les questions liées aux temps de vie et à la mobilité professionnelle, la visibilité et les " risques " qui lui sont associés dans certains postes où elles demeurent en minorité. On peut se demander si ces perceptions ou auto-perceptions stéréotypées comme les contextes organisationnels sont des facteurs mis en avant dans le discours des personnels de direction notamment chez les femmes. 


\subsection{Perceptions des inégalités par les femmes}

Des recherches ont été menées pour étudier la façon dont les inégalités de sexe sont perçues par les femmes et les hommes. Analysant la perception d'une douzaine d'inégalités (revenus, patrimoine, emploi, etc.), l'étude de Galland, Lemel, et Frénod (2013) montre que les inégalités de sexe font partie de celles qui sont considérées comme les plus illégitimes lorsqu'elles sont appréhendées globalement pour la société. En revanche, les personnes interrogées se sentent moins directement concernées à titre personnel par ce type d'inégalités. Ce résultat est corroboré par l'étude de Richardot, Collet, et Frénod (2016). Prenant appui sur l'enquête Dynegal, les trois auteures montrent que «si les femmes se montrent plus sensibles aux inégalités en général, elles semblent éprouver des difficultés à exprimer ou ressentir les injustices qui les touchent personnellement " (Richardot et al., 2016, p. 96). Interrogées sur la question des inégalités de sexe, certaines femmes évoquent un type d'argumentation qui s'inscrit dans le registre de la naturalisation. Le recours à ce genre de justification tire sa force de son caractère « d'évidence " en se référant à des caractéristiques biologiques « incontestables ». C'est ce que montre l'étude qualitative de Sénac-Slawinski (2007). Analysant finement les représentations de quarante-six personnes ayant des profils sociodémographiques contrastés, elle montre comment cet " ordre naturel " s'élabore en articulation étroite avec "l'ordre social et politique ". L'analyse de la perception des inégalités révèle que certaines femmes semblent avoir intériorisé leurs positions dominées et s'en montrent "satisfaites ". Ce type de constat peut être mis en lien en psychologie sociale avec la théorie de la justification du système (Jost \& Banaji, 1994) qui postule que les individus ont tendance à préférer trouver des raisons aux injustices et déséquilibres d'un système plutôt que de le remettre en cause. Cette théorie décrit et explique les mécanismes psychosociaux par lesquels un système social maintient un certain statu quo entre les groupes, fondé sur un rapport de domination et sur des processus qui légitiment ces rapports de domination. Selon la théorie de la justification du système (Jost \& Banaji, 1994), les stéréotypes sociaux peuvent être considérés comme des outils de légitimation du système. Ainsi, l'adhésion aux stéréotypes de genre, de même que l'auto-stéréotypie renforce la justification du système chez les femmes exposées à une situation d'inégalité défavorable à leur propre groupe (Verniers \& Martinot, 2015).

\subsection{Inégalités perçues concernant les rémunérations et la conciliation des temps de vie}

Il s'agit notamment de la perception des inégalités objectives qui concernent les salaires et le partage des tâches domestiques. Ainsi, à temps de travail et métiers équivalents, les femmes touchent 12,8 \% de moins que les hommes (Observatoire des inégalités, 2019). Prenant appui sur une enquête de l'INSEE réalisée auprès d'un échantillon représentatif de 6000 personnes, Baudelot et Serre (2006) montrent que les femmes se déclarent cependant aussi satisfaites que les hommes de leurs rémunérations tout en ayant des salaires inférieurs. Les hommes auraient tendance à se comparer à des collègues mieux payés alors que les femmes se comparent à leurs collègues moins bien rémunérées. Les femmes comparent également leurs situations avec celles des femmes des générations précédentes beaucoup plus marquées par les inégalités professionnelles et salariales (Marry, 2014). Nous pourrions penser que ceci ne concerne pas les professions prestigieuses pour lesquelles le haut niveau de diplôme serait susceptible de garantir une égalité salariale. Une enquête réalisée auprès de plusieurs promotions de polytechniciens et polytechniciennes montre que ce n'est pas le cas (Marry, 2014). Les entretiens réalisés révèlent que les différences de salaires sont clairement perçues par les personnes interrogées mais ces dernières n'estiment pas qu'il s'agisse d'inégalités injustes.

Nous observons le même type de résultats au sujet du partage des tâches domestiques. La littérature existante montre que dans ce domaine-là, les inégalités objectives restent encore très marquées : les femmes assureraient 33,2 heures de travail hebdomadaire contre seulement 18,2 heures pour les hommes, soit près du simple au double (Singly et de Singly, 2007, p. 37). "L'incidence objective de la vie familiale et conjugale sur la vie professionnelle est en effet inégale entre les femmes et les hommes, et c'est d'abord et avant tout aux femmes que la conciliation "pose problème" " (Marry et al., 2015, p. 59). Malgré ce déséquilibre, près des deux tiers des femmes estiment que ce partage des tâches est juste. Singly et de Singly (2007) montre que les femmes ont 
recours à des critères différents d'investissement dans les tâches ménagères que ceux des hommes. Mais cette appréciation asymétrique des situations concourt à la reproduction des inégalités. L'auteur souligne la prégnance des modèles d'identification sexués traditionnels qui pèsent encore lourdement sur l'orientation de nos comportements. Les évolutions sociétales récentes ont bien sûr contribué au fait que les normes de sexe et de genre soient "transgressées " mais les travaux qui ont analysé ce phénomène révèlent que le " prix à payer " pour les femmes demeure encore très élevé et parfois dissuasif (voir notamment les recherches qui ont convoqué le concept d'inversion du genre [GuichardClaudic, Kergoat, \& Vilbrod, 2008]).

\section{Objectifs}

Cette recherche exploratoire vise à étudier comment les personnes (et particulièrement les femmes) occupant des postes à responsabilité du second degré perçoivent les inégalités genrées : les percoivent-ils.elles ou non ? Si oui, quelles en sont les explications ? Quels sont les facteurs identifiés au niveau du parcours professionnel ? En effet, Marry et al. (2015) ont montré dans leur étude une euphémisation, "voire une négation des inégalités sexuées dans la fonction publique de la part des femmes comme des hommes, malgré le maintien, attesté et mesuré, de ces inégalités et en dépit des expériences par ailleurs vécues ou rapportées par les enquêtés. » (Marry et al., 2015, p. 62). Allons-nous retrouver ce déni ou, au contraire, un regard porté à l'aune du genre ?

Pour répondre à ces questions nous avons adopté une méthodologie mixte. L'étude a été réalisée en deux temps. Lors de la première phase, une enquête nationale par questionnaire a été menée. Les caractéristiques se celle-ci sont présentées ci-dessous. Lors de la deuxième phase, nous avons interviewé 25 chefs d'établissement à deux périodes différentes (2010 et 2019) afin de repérer les évolutions qui auraient pu apparaître dans le laps de temps.

\subsection{Design et méthodologie}

\subsubsection{Données questionnaire national}

Nous avons ré-exploité certaines données issues d'une enquête nationale que nous avons menée en 2010 auprès de chef.fes d'établissement. Parmi les matériaux disponibles, nous avons sélectionné les réponses à une question ouverte qui concernait, à l'époque de l'enquête (2010), le pourcentage de femmes cheffes d'établissement (43,8\%), pourcentage qui a évolué depuis (48,9\% en 2017). " les statistiques nationales révèlent qu'en janvier 2009, 43,8\% des personnels de direction du second degré sont des femmes. Selon vous, quelles sont les raisons qui expliquent cette représentation inégale des femmes et des hommes dans la fonction ? (Développer la réponse) ». Les réponses fournies par les personnes interrogées permettent d'identifier indirectement la façon dont sont perçues les inégalités éventuelles dans l'accès aux postes de direction au regard du genre. Comme le soulignent Marry et al. (2015), les réponses indiquent-elles une négation des inégalités sexuées ou une prise de conscience de dynamiques genrées intervenant dans le processus d'accès aux responsabilités de direction ?

Nous avons obtenu 292 réponses qui se présentent sous la forme de développements écrits allant de une à quinze lignes. Elles ont fait l'objet d'une analyse de contenu thématique telle qu'elle est préconisée par Bardin (2001). Pour être en mesure d'analyser la totalité du matériau recueilli (un peu plus de mille lignes dactylographiées), nous avons élaboré 34 catégories regroupées en huit rubriques. Ces dernières sont consultables dans le tableau consigné en Annexe. La représentativité de notre échantillon est satisfaisante eu égard aux caractéristiques habituellement prises en considération : le sexe, l'âge, les qualifications académiques, la répartition selon le type d'établissement (Cacouault \& Combaz, 2011).

\subsubsection{Entretiens semi-directifs}

En articulation étroite avec cette démarche quantitative, vingt-cinq entretiens ont été réalisés. Pour des raisons de commodité d'accès, ils ont été menés dans trois régions distinctes : Rhône-Alpes, le Limousin et la région parisienne. Ces vingt-cinq entretiens ont été menés lors de deux campagnes : quinze en 2010 et dix en 2018-2019. L'objectif était de déceler d'éventuelles évolutions affectant la 
Tableau 1

Caractéristiques des personnes interviewées.

\begin{tabular}{llllll}
\hline Sexe & Moyenne d'âge & Proviseur.e & Proviseure adjointe.e & Principal.e & Principal.e adjointe.e \\
\hline Femmes & 48,3 ans & 3 & 4 & 3 & 3 \\
Hommes & 46,7 ans & 4 & 2 & 5 & 1 \\
\hline
\end{tabular}

façon dont les inégalités sont appréhendées entre les deux dates. La population interrogée est caractérisée par des profils diversifiés avec cependant une sur représentation des hommes dans des postes de direction (proviseur.e.s de lycées et principaux et principales de collèges [Tableau 1]).

Au cours des entretiens, les personnes interviewées ont été invitées à retracer précisément leurs parcours professionnels et la façon dont ces derniers pouvaient s'articuler aux autres temps de vie, en particulier l'engagement dans les responsabilités familiales. Par ailleurs, les interviewé.e.s ont été incité à commenter la statistique $(43,8$ \% en 2009) relative à l'accès aux postes de personnels de direction. Les entretiens ont fait l'objet d'une analyse de contenu qui reprend les catégories élaborées pour l'étude des réponses à la question ouverte.

\section{Les résultats}

\subsection{Enquête nationale par questionnaire}

Pour l'enquête nationale, la répartition des réponses a été étudiée en fonction des différentes variables prises en considération : le sexe, l'âge, le type de poste occupé (proviseur, proviseur adjoint, etc.), le niveau de diplôme, l'origine sociale, le statut familial (célibataire, marié, divorcé, etc.), le nombre d'enfants. Pour chaque tableau croisé, la validité statistique des écarts a été vérifiée avec un test du $\mathrm{Chi}^{2}$. Seules les différences en fonction du sexe sont significatives. La présentation des tendances chiffrées s'accompagne d'analyses qui prennent appui sur des extraits de réponses. Ceci permet d'apprécier finement la nature des formulations choisies et le type d'argumentation mobilisé.

\subsubsection{Les non-réponses à la question : du déni?}

En premier lieu, il convient de s'intéresser aux $12 \%$ de personnes qui n'ont pas répondu à la question posée dans le cadre de l'enquête nationale. Il s'agit là d'un résultat en soi qu'il ne faut pas négliger. Les personnes interrogées ont pu considérer que cette question était " gênante » ou qu'elle n'avait pas lieu d'être posée. Parmi ceux et celles qui n'ont pas répondu, les hommes sont deux fois plus nombreux que les femmes $\left(15,0 \%\right.$ contre $\left.6,3 \%, \mathrm{Chi}^{2}=10,2, p<.01\right)$. Une minorité de personnes $(2,4 \%)$ a précisé qu'elles n'avaient aucune opinion par rapport la question. Ce sont tous des hommes comme ce principal de collège âgé de 60 ans qui répond : "Je suis un homme, je ne sais pas ". Ce type de réponse peut paraître surprenant car tous les chefs d'établissement ont pris connaissance des différentes conventions interministérielles qui ont été signées pour tendre vers une meilleure égalité entre les filles et les garçons et entre les femmes et les hommes au sein du système éducatif. Bien sûr, il ne s'agit que d'une minorité d'hommes mais leurs réponses s'apparentent à une forme de déni des inégalités de sexe. Nous reviendrons un peu plus loin sur cet aspect puisqu'une partie des hommes qui ont répondu conteste la validité de la statistique évoquée dans la question suivante (pour rappel : $43,8 \%$ des personnels de direction du second degré sont des femmes).

\subsubsection{Les interprétations avancées pour commenter la statistique : inégalités, temps de vie et caractéristiques genrées de la fonction}

3.1.2.1. Des réponses relatives à l'articulation entre vie professionnelle et vie personnelle. La répartition des réponses montrent que celles qui sont de loin les plus nombreuses (évoquées par 53,5\% des chef.fe.s d'établissement), concernent les difficultés pour " concilier » l'engagement professionnel, les responsabilités familiales et la vie personnelle. Elles sont plus souvent mentionnées par les femmes 
(60,1 \% contre 47,3\% pour les hommes, $\left.\mathrm{Chi}^{2}=8,89, p<.05\right)$. Malgré cette différence d'appréciation mise au jour quantitativement, des points de convergence apparaissent lorsqu'il s'agit de souligner l'incompatibilité entre, d'une part, la grande disponibilité que requiert le poste de direction et la charge de travail qui est associée et, d'autre part, les contraintes liées à l'éducation des enfants et aux tâches ménagères. Certaines femmes précisent qu'elles donnent la priorité à leur famille : "Cette fonction est très prenante et je pense que la plupart des femmes préfèrent privilégier leur vie de famille, notamment leurs enfants. Moi-même, si j'avais vraiment mesuré le temps passé au travail, je n'aurais peut-être pas passé le concours » (proviseure adjointe, 43 ans). D'autres indiquent qu'elles ont pu embrasser la carrière de chefs d'établissement grâce au soutien de leur conjoint : « Je pense que les femmes sont conscientes de l'investissement personnel que demande cette fonction. Elles mènent souvent une vie familiale intense en parallèle et attendent donc d'être plus disponibles pour intégrer cette catégorie de personnels. Pour ma part, mes enfants ont 6 et 9 ans et mon conjoint participe pleinement à l'organisation familiale en plus de sa carrière professionnelle. C'est pour cela que j'ai pu mener à bien ce projet » (principale adjointe, 39 ans). Même si c'est beaucoup plus rare, plusieurs hommes le mentionnent également. Ainsi, ce proviseur de lycée professionnel âgé de 63 ans précise : " Pour s'occuper de nos trois enfants, ma femme s'est mise en disponibilité à partir du moment où j'ai assumé des responsabilités (d'abord en faisant fonction d'inspecteur du second degré puis en tant que personnel de direction). L'évolution de carrière de mon épouse a donc pâti de la mienne. Si elle n'avait pas assuré (avec succès) l'éducation de nos enfants, je n'aurais pas pu envisager de prendre un poste de direction ». Une minorité d'hommes se montre plus réservée sur la question de la disponibilité des femmes : "La fonction est à la portée de tout un chacun, il suffit juste d'être disponible et mobile. Les femmes le sont-elles moins ? " (proviseur adjoint, 54 ans). Comme nous l'avons déjà souligné plus haut, tout se passe comme si un certain nombre de femmes attendaient d'être déchargées des tâches familiales pour accéder aux postes de direction. C'est le cas notamment lorsqu'elles sont divorcées ou séparées. C'est ce qu'évoque cette principale adjointe de 45 ans : "Compte tenu de l'aspect chronophage de la fonction, elle est difficilement compatible avec une vie de couple et de famille. Dans ma formation, en 2006, nous étions 9 femmes dont 7 étaient divorcées ou séparées (8 avec moi désormais !)".

3.1.2.2. Des raisons données en lien avec les caractéristiques intrinsèques de la fonction. Dans l'ordre décroissant d'importance, la deuxième raison évoquée pour expliquer le moindre accès des femmes aux postes de direction tient aux caractéristiques intrinsèques de la fonction (grande disponibilité, charge de travail très lourde, obligation de mobilité géographique, etc.). Plus de la moitié des personnes $(52,9 \%)$ font référence à ces dimensions, les femmes un peu plus souvent que les hommes ( $57 \%$ contre $49,1 \%$ pour les hommes, $\mathrm{Chi}^{2}=7,08, p<.05$ ). Pour certaines dirigeantes, il est préférable d'être libérées des contraintes familiales pour accéder à la fonction : « La grande disponibilité qu'exige cette fonction nécessite d'être "libérée" d'obligations familiales. Il faut accepter d'être moins disponible pour sa famille, ses enfants. Je pense que c'est possible quand ils atteignent l'adolescence, voire après..." (principale adjointe de collège, 41 ans). L'obligation de mobilité géographique représente aussi un obstacle important qui ne semble pas être appréhendée de la même manière par les hommes et les femmes. Ainsi, cette proviseure adjointe de 48 ans mentionne que "La société française fonctionne encore sur de vieux schémas. Dans le jeu des mutations, s'il est difficile pour un homme d'entrainer sa famille dans un changement radical de vie (rythme de travail, ville ou région, logement de fonction) ; pour une femme, cela semble souvent impensable ". En lien avec les caractéristiques familiales, les contraintes matérielles liées à l'obligation de résider dans l'établissement scolaire sont également évoquées : "En plus de la contrainte de mobilité géographique, il y a aussi la question du logement dont la surface n'est pas toujours adaptée à la taille de la famille (proviseure, 55 ans). Dans certains cas, la charge de travail et la grande disponibilité sont mises en balance avec la rémunération et le prestige considérés comme très insuffisants. Et ceci pourrait expliquer le désintérêt progressif des hommes pour la carrière de chef d'établissement : "Ce sont des professions dont les hommes ne "veulent" plus et qui donc demeurent plus facilement accessibles aux femmes : beaucoup de travail, peu de reconnaissance morale, de moins en moins de "prestige" et des rémunérations qui ne sont pas à la hauteur des heures passées à travailler " (proviseur de lycée, 41 ans). 
3.1.2.3. Des réponses en lien avec les stéréotypes de genre, la socialisation genrée et le pouvoir. Proportionnellement moins fréquentes (15,6\%), apparaissent les réponses qui soulignent le poids des attentes sociales vis-à-vis de l'accès des femmes et des hommes aux postes de direction et le fait que la division sexuée du travail soit encore très marquée au sein de notre société. Les dirigeantes le mentionnent un peu plus souvent que leurs homologues masculins (19\% contre $12,4 \%$, Chi ${ }^{2}=8,4$, $p<.05)$. Les qualités « naturelles » des unes et des autres constituent un argument classique sensé faire autorité. Celui-ci semble parfaitement intériorisé par quelques femmes de notre échantillon comme l'illustre l'extrait suivant : " Il me semble qu'historiquement, les postes dit "à pouvoir" nécessitant des prises de décisions ont toujours naturellement favorisé les hommes. Socialement, on fait peu confiance aux femmes sur la prise de décision et la direction en général. Il est naturel que, dans la fonction publique, cela soit identique " (principale adjointe de collègue, 42 ans). L'importance de l'éducation dans le façonnage des modèles d'identification sexuée est également souligné : "La société est plus habituée à être dirigée par des hommes et certaines femmes marquées par leur éducation, leur entourage, n'osent pas franchir le pas" (proviseure, 60 ans). La hiérarchie est parfois mise en cause comme n'hésite pas à le faire cette proviseure de 57 ans. Elle souligne l'importance " du regard des autres. . qui s'attendent davantage à ce que le chef soit un homme. Combien de fois ils se sont adressés devant moi à mon adjoint comme s'il était le proviseur ! Et le propos tenu par un recteur à un proviseur : "pour ce poste-là, il faut un homme de belle portance, comme monsieur Untel". Ceci est très significatif : il y a encore beaucoup de retard dans les représentations ... et donc il faut plus d'audace à une femme pour se lancer ». D'autres se plaignent des remarques désobligeantes de leurs collègues masculins : « Le monde de l'Éducation nationale, au niveau des chefs d'établissements, est très machiste. J'ai personnellement été témoin d'attitudes méprisante de proviseurs hommes, se mettant à plusieurs pour se moquer d'une collègue au prétexte que ce poste était sans doute trop "difficile" pour une femme et ce, sans complexe, devant moi qui ne suis qu'adjointe et... femme " (principale adjointe de 53 ans).

Certaines caractéristiques individuelles sont mentionnées pour expliquer la plus faible représentation des femmes aux postes de chefs d'établissement. L'ensemble représente 9,2\% des réponses et elles sont mentionnées plus souvent par les femmes ( $12,7 \%$ contre $5,9 \%$ pour les hommes, $\mathrm{Chi}^{2}=10,18, p<.01$ ). Ainsi, les femmes seraient moins ambitieuses que les hommes, elles auraient moins confiance en elles, elles se sous-estimeraient, elles s'autocensureraient davantage. Les réponses des hommes sont moins nombreuses mais elles renvoient plus fréquemment aux attitudes d'autocensure des femmes : "Les femmes osent moins que les hommes demander à accéder à des fonctions de direction ou en général à des fonctions de responsabilité. Elles se croient (encore et à tort !) moins capables que les hommes d'avoir de l'autorité " (principal adjoint de collège, 61 ans). Le niveau d'exigence pour envisager la carrière de dirigeant serait plus élevé chez les femmes et ces dernières seraient plus réalistes dans l'appréhension des charges de travail, comme le suggère ce principal adjoint de collège âgé de 61 ans : "Plus de perfectionnisme chez les femmes, plus de projections dans "le faire" et non dans "le dire" peuvent être des freins à ce grand saut ".

Un autre chef d'établissement déplore le manque de soutien de l'institution vis-à-vis des femmes : " Pour moi, les femmes se censurent elles-mêmes et le manque de structure de ressources humaines pour proposer, accompagner les perspectives de carrière sont aussi un frein " (proviseur adjoint, 60 ans).

Les caractéristiques individuelles des hommes sont beaucoup moins souvent évoquées (3,7 \%). Certaines "spécificités " masculines sont citées : plus ambitieux, plus sensibles à la reconnaissance sociale associée au poste de direction, plus attirés que les femmes par le pouvoir. Ainsi, ce proviseur de lycée professionnel âgé de 54 ans souligne " la plus forte ambition des hommes qui recherchent davantage la reconnaissance professionnelle (aiment être mis en valeur par leur hiérarchie) et une reconnaissance financière ". Aussi classique qu'il puisse paraître, ce type d'argumentation ne suscite pas nécessairement une adhésion pleine et entière de toutes les personnes qui ont fourni une réponse. Certaines se montrent prudentes et circonspectes : " J'interprète cette situation avec beaucoup de prudence, comme la résultante d'un goût prononcé des hommes pour l'exercice d'un pouvoir fut-il dérisoire et limité » (principal de collège âgé de 39 ans).

\subsubsection{Une minorité qui ne perçoit pas d'inégalités}

Outre les réponses qui viennent d'être présentées, la question ouverte a suscité des réactions plus ou moins vives vis-à-vis du pourcentage évoqué (pour rappel $43,8 \%$ de femmes qui sont chef 
d'établissement en 2009). Une minorité (6,4\%) conteste la validité de cette statistique nationale soit pour relativiser l'ampleur de l'inégalité, soit en contester l'existence (les hommes un peu plus souvent que les femmes : 7,7 \% contre $5,1 \%, \mathrm{Chi}^{2}=8,31, p<.05$ ). Ce sont les formulations et les arguments utilisés qui distinguent le plus les deux sexes. Les propos les plus directs et les plus catégoriques sont portés par des hommes. Ainsi peut-on lire sous leur plume : " $43,8 \%=$ presque la moitié, je ne vois donc pas où est l'inégalité ! " (principal de collège, 58 ans). " Presque $45 \%$ ne me semblent pas créer une réelle inégalité - on est proche des $50 \%$ » (proviseur de lycée, 60 ans). Certains se montrent irrités par la question et ils suggèrent que l'écart entre les femmes et les hommes va progressivement se réduire : "Cela ne me semble pas un problème qui mérite une attention particulière d'autant que progressivement cet écart diminue. Dans moins de 10 ans, il y aura beaucoup plus de personnels de direction de sexe féminin que de sexe masculin ! C'est déjà le cas chez les avocats, les médecins, les enseignants, le personnel soignant des hôpitaux... Que l'on arrête enfin de se focaliser sur un problème qui n'en est pas un " (proviseur de lycée professionnel, 54 ans). Pour d'autres, l'accès à la direction d'établissement relève avant tout de qualités personnelles et ne doit pas faire l'objet de quotas imposés : "On voudrait idéalement pour compenser un déficit fonctionnel historique qu'il y ait $50 \%$ de femmes et $50 \%$ d'hommes à des postes de direction. Il ne s'agit pas d'une affaire de quotas. La direction est surtout une question de tempérament. Le concours est ouvert à tous. Alors, où se situe le problème réellement ? "(proviseur adjoint de lycée, 35 ans). Comparativement aux hommes, les femmes qui se sont exprimé ne remettent pas fondamentalement en cause la statistique évoquée. Elles se montrent plus nuancées et toutes indiquent que l'on tend vers l'égalité. L'une d'entre elles estime que les femmes sont plutôt bien représentées : "Aucune raison particulière. Je trouve, qu'au contraire, les femmes sont bien représentées " (principale de collège, 47 ans).

\subsubsection{Des inégalités "cachées ", des biais dans le recrutement et une perspective d'évolution}

Quelque peu à rebours de ce que nous venons de voir, la validité de la statistique évoquée est remise en cause pour son caractère trop globalisant (5,8\% des répondants). Elle masque les véritables inégalités. Celles-ci sont sans doute beaucoup plus fortes lorsqu'on affine l'analyse en prenant en considération la nature du poste. C'est ce que mentionnent précisément deux proviseures de lycées âgées de 63 ans : "Certes 43,8\% sont des femmes mais en affinant un peu... on retrouve les inégalités récurrentes. Représentées dans les postes d'adjoints et sur des collèges. De fait, sur les lycées et, qui plus est, sur les " gros " établissements, peu de femmes sont choisies ! ". " les femmes sont moins représentées que les hommes dans les fonctions de direction mais il faut affiner les statistiques : nombre de femmes ayant des postes de lycées de $4^{\mathrm{e}}$ catégorie ou plus ? ... avec un peu de chance, ce sont des principales... et des adjointes au lycée. Les postes sont ciblés ! ”.

Une partie des personnes interrogées $(6,1 \%)$ suggère qu'une évolution est en cours allant dans le sens d'une répartition plus équilibrée en fonction du sexe. La principale raison évoquée est le fait que le principal vivier de recrutement est constitué des enseignants du second degré. Ce dernier étant très féminisé, la part des femmes devrait augmenter progressivement. Pour compléter ce constat qui est confirmé par les dernières statistiques disponibles, certains ajoutent que de plus en plus de femmes se présentent au concours de recrutement et que cela contribue à rétablir l'équilibre : "Cela va changer car, au concours, de plus en plus de femmes sont présentes et le réussissent. La "barrière" des contraintes d'emploi du temps est moins mal vécue par les femmes (des collègues de promo sont aussi jeune mamans). Si vous regardez ces mêmes statistiques pour les personnels de direction de moins de 45 ans, elles doivent s'inverser ! " (principal adjoint, 40 ans).

Une faible minorité de chef.fe.s d'établissement $(2,8 \%)$ souligne la responsabilité de l'institution en évoquant les biais dans les procédures de recrutement : « Il n'y a pas de parité au concours. Le mode de sélection repose sur un système très archaïque, basé sur la cooptation par une hiérarchie peu féminisée " (principal adjoint, 39 ans).

\subsection{Entretiens semi-directifs}

Les tendances mises au jour à partir de ces matériaux qualitatifs corroborent en grande partie ce qui a été montré précédemment à partir de l'enquête nationale par questionnaire. Ceci apparaît clairement lorsqu'il s'agit d'évoquer les difficultés à concilier l'engagement professionnel et les 
responsabilités familiales ainsi que le poids des stéréotypes sexués. Mais, au-delà de ces points communs, les entretiens permettent de cerner plus finement comment chaque personne interrogée se situe personnellement par rapport à la question de l'inégalité d'accès aux postes de direction. Les perspectives d'évolution sont également bien plus développées, en particulier celles qui sont associées à la succession des différentes générations. Nous assistons depuis plusieurs années à une transformation du rapport que les plus jeunes entretiennent au travail et ceci semble encourager l'ambition professionnelle d'une partie des femmes que nous avons eu l'occasion d'interroger.

Les propos tenus par les personnes interviewées n'ont quasiment pas varié entre 2010 et 2019.

\subsubsection{Influence du genre sur son parcours professionnel : déni en majorité}

L'enquête complémentaire par entretiens semi-directifs $(n=25)$ a permis d'interroger les chefs d'établissement à propos de la façon dont ils pouvaient appréhender la question des inégalités d'accès à la fonction en prenant en considération leurs propres parcours professionnels. Seules trois personnes, deux femmes et un homme (sur vingt-cinq) ont fourni quelques éléments de réponse. Ces derniers révèlent le décalage - déjà mis au jour par certains travaux évoqués en introduction - entre le type d'analyse proposé lorsqu'il s'agit d'évoquer les inégalités sexuées de façon générale et, par ailleurs, la manière dont la question peut se poser pour son cas particulier. Les formulations peuvent s'avérer très brèves, comme s'il fallait se débarrasser de cette question : "Cela n'a pas du tout influencé mon parcours, c'est un argument que je n'ai jamais évoqué ni même imaginé " (principal de collège, 55 ans). Ce positionnement n'est pas l'apanage des hommes : «ça, c'est une colle... je ne sais pas. Moi je ne me suis jamais posé la question en fait, homme-femme dans la fonction " (proviseure, 52 ans). Il est sans doute difficile de porter un regard rétrospectif sur son propre parcours comme le suggèrent les propos tenus par cette proviseure de 63 ans : "C'est vrai que c'est une question que je me suis jamais vraiment posée, c'est plutôt un cheminement personnel. Mais cela dépend aussi du contexte, des hommes avec lesquels on peut travailler. Il y a des hommes qui vont reconnaître les compétences féminines, et d'autres qui vont être complètement dans le refus, c'est clair ». Quelques mots prononcés par cette femme suggèrent qu'elle aurait pu intérioriser son incompétence : " Je pense qu'il y a eu inconsciemment l'idée que je n'étais pas capable et c'est vrai qu'il faut un peu se forcer. Je pense que beaucoup de femmes sont dans cette démarche-là, c'est pour ça que je dis aux jeunes femmes "allez-y, ne vous posez pas de questions, les garçons ne se posent pas de questions, vous, faites la même chose" ".

Les recherches menées sur l'introduction des études centrées sur le genre et sur les inégalités sexuées dans le cadre de la formation des enseignants montrent toutes les difficultés et les réticences liées au fait de se situer personnellement en tant que femme ou homme par rapport à ces questions. Le travail de prise de conscience et de réflexivité que cette démarche implique à tendance à bousculer l'identité sexuée de chacun entraînant des résistances très fortes (Léchenet et al., 2016).

Les tendances mises au jour par nos entretiens montrent que la théorie de la justification du système évoquée plus haut paraît très pertinente. Pour interpréter nos résultats, la référence aux travaux sociologiques centrés sur la domination peut également être mobilisée. À cet égard, les écrits de Max Weber (1921) montrent que les mécanismes de domination fonctionnent de manière d'autant plus efficace que les acteurs sociaux sont " consentants".

À présent, il importe de présenter les évolutions qui sont perçues par les chefs d'établissement interrogés ainsi que les obstacles qui demeurent.

\subsubsection{Des évolutions perçues?}

Les données recueillies par entretiens et des statistiques nationales récentes (MEN, 2018) laissent penser qu'une évolution se dessine avec les générations les plus jeunes. Les femmes sont actuellement plus nombreuses que les hommes à se présenter au cours de recrutement des personnels de direction. Elles n'attendent pas, comme leurs aînées, d'être déchargées de leurs responsabilités familiales pour faire acte de candidature. Parmi les générations les plus jeunes et les plus diplômées, le rapport au travail évolue. Les jeunes hommes sont plus présents dans leurs familles et ils sont plus impliqués dans les soins qu'ils donnent à leurs enfants (Méda \& Vendramin, 2010). L'épanouissement au travail s'avère primordial à condition que cela n'empiète pas trop sur la sphère familiale. Le plaisir éprouvé dans l'exercice de l'activité professionnelle peut avoir des conséquences positives sur la vie familiale 
comme le suggère cette principale de 44 ans : " on n'a pas attendu que les enfants soient grands parce que le principe de vie était de dire qu'il faut que chacun soit heureux avant tout. Et, si on est chacun heureux dans son métier, qu'on arrive à s'épanouir, on sera aussi heureux à la maison et les enfants en profiteront, plutôt que d'attendre 10 ou 15 ans en n'étant pas trop épanoui. C'était le parti pris. Je ne sais si on avait raison mais, en tous les cas, c'était le nôtre ".

Parmi les couples jeunes et diplômés, la double carrière menée simultanément paraît représenter une situation équilibrée. La carrière de l'un ne devant pas se dérouler au détriment de celle du conjoint : "Avec mon époux, on s'est tout de suite mis d'accord sur le fait que les deux carrières étaient importantes et intéressantes et qu'aucun d'entre nous ne devait se mettre en retrait ou vivre dans l'ombre de l'autre " (principale de collège, 42 ans).

Les femmes les plus jeunes semblent plus ambitieuses que leurs collègues plus âgées mais elles ne négligent pas pour autant les arbitrages qu'il faudra probablement opérer entre l'engagement professionnel et les responsabilités familiales. Par ailleurs, elles n'ignorent pas les difficultés auxquelles elles risquent d'être confrontées en tant que chef d'établissement. Malgré cela, une partie d'entre elles aspire à franchir les différentes étapes de la carrière et ne souhaite pas rester dans une poste d'adjointe : « Pour moi, adjointe ce n'est qu'une étape. Je crois que si je n'allais pas au bout du cheminement, je serais déçue. Je me vois mal rester qu'adjointe, ça n'est pas possible pour moi. Comment je l'explique? Est-ce que c'est justement les charges familiales qui font que... ? (rires) Je pense qu'il doit y avoir beaucoup de ça même si, par ailleurs, mes collègues de la promo où je suis n'ont pas du tout envie de rester adjointes. Dans la promo où on était avec S., je n'ai jamais entendu quelqu'un espérer rester adjointe toute sa vie. Donc maintenant, est-ce que ce n'est pas une question de génération, je ne sais pas. C'est vrai que ça fait peur, on craint un peu le fait d'être seule à bord mais, bon, d'autres y sont passés et ils y arrivent très bien donc pourquoi pas nous ? Et on n'est pas plus bêtes que les autres et, voilà, en même temps, peut être que les femmes se mettent des barrières aussi dans leurs têtes... Chose que moi je n'ai pas. Moi, je ne dirais pas 'c'est un truc d'homme', pour moi je peux accéder à tous les postes si tant est que j'en ai les qualités et les compétences (principale adjointe, 44 ans).

L'évolution du contexte sociétal est également évoquée pour montrer comment les transformations en cours peuvent inciter une partie des femmes à s'engager davantage dans la sphère professionnelle. La répartition des tâches familiales évolue graduellement et les aides institutionnelles se développent : " Je pense que c'est peut-être culturel, sociétal. Les femmes s'autorisent, elles, davantage à s'exprimer dans le monde professionnel. Il y a une meilleure répartition peut-être des tâches ou des missions entre hommes et femmes ; davantage d'hommes osent aussi prendre en charge et s'occuper des enfants. Il y a également de la discrimination positive dans le cadre du concours de recrutement. Et, puis j'étais en train de réfléchir s'il n'y avait pas davantage de mode de garde ou de solution de garde pour les enfants en France » (principale de collège, 45 ans).

Les évolutions perçues par les personnels de direction interviewés concernent également les transformations qui ont modifié le rôle qu'ils jouent au sein des établissements scolaires. Cela touche en particulier le rôle pédagogique qui a été officiellement institué en France par le référentiel de 2000. C'est l'hypothèse qui est avancée par ce proviseur de 44 ans : " peut-être que les femmes ont arrêté de se censurer et peut être qu'elles ont vu la fonction différemment. Au lieu de voir une fonction où, il y a 20 ans, il fallait avoir de l'autorité et il fallait en imposer, on est arrivé à une fonction où il faut établir une certaine forme de consensus, de regroupement autour d'un projet et, peut-être que làdessus, les femmes s'y retrouvent un peu plus. Elles se retrouvent plus dans la fonction de personnels de direction parce qu'il est peut-être plus là pour former une équipe, l'animer et pour être un pédagogue. J'ai l'impression que les femmes ont une sensibilité un petit peu plus fine par rapport à la pédagogie, c'est peut-être pour ça qu'on les retrouve plus dans le monde enseignant. Cette relation à la pédagogie et l'aspiration à faire consensus autour d'un projet, c'est pour ma part plus typiquement féminin ".

Les évolutions qui viennent d'être évoquées ne sont pas négligeables mais elles ne doivent pas occulter un certain nombre de résistances persistantes.

\subsubsection{Des résistances qui demeurent?}

L'un des proviseurs que nous avons interrogé souligne le contraste existant entre le manque d'ambition des femmes et le caractère décomplexé des hommes : "le manque d'ambition parce que 
chez les femmes, comme dans tous les secteurs professionnels, on craint de ne pas savoir faire. Les femmes ne font que quand elles savent parfaitement faire. Nous [les hommes], on fait semblant de faire dans n'importe quelle situation. Ma propre femme qui a passé le concours [de personnels de direction] il y a très peu de temps, m'a dit "mais je ne saurais pas faire, je n'ai pas essayé ; je ne saurais pas faire". Alors qu'un mec, il dit "je ne sais pas faire mais je m'en fous, je vais y aller et on verra bien" ". (...) Le mec il ne connaît pas bien ses compétences mais il les estime toujours plus importantes que ce qu'elles sont, donc il vise plus haut au risque de se casser la figure (proviseur de 59 ans).

Certains stéréotypes semblent encore très présents, notamment ceux qui sont relatifs aux capacités supposées des hommes et des femmes à assumer des responsabilités importantes comme, par exemple, le fait de diriger des établissements scolaires de taille importante : " on accorde plus de crédit à un homme qu'à une femme. La femme, elle doit prouver qu'elle peut assumer tel poste, par exemple, diriger un plus gros établissement ; elle doit prouver, tandis que l'homme, on part du principe qu'automatiquement, il va y arriver et ça, c'est bien ancré. Comme si c'était naturel qu'à la tête d'un gros établissement, il faut un homme et pas une femme. À partir du moment où vous êtes homme, vous n'avez pas besoin de prouver vos compétences. On sait que, par définition, vous allez pouvoir le faire " (principale adjointe, 57 ans).

La question de la confiance en soi semble encore représenter un élément qui limite l'ambition d'une partie des femmes interrogées : "Je pense qu'il y a une différence de mentalité entre les hommes et les femmes ou une partie des hommes et des femmes ; c'est-à-dire que la femme, à niveau de compétence, de formation et d'expériences équivalentes n'osera peut-être moins postuler qu'un homme. Moi, je le perçois dans mes fréquentations personnelles et professionnelles où les femmes sont nettement plus hésitantes car elles manquent de confiance en elles. Alors qu'un homme qui n'a pas plus de compétences ou d'expérience ne se posera pas la question de "est ce que je suis compétent pour ?", la femme va se la poser " (principale de collège, 44 ans).

Au cours des entretiens que nous avons menés, certains chefs d'établissement suggèrent que les femmes seraient moins "disposées " à entretenir des relations conflictuelles qui ne manquent pas d'apparaître lorsqu'on exerce des fonctions de direction. L'un des principaux de collège interrogés précise que les femmes se sentiraient plus impliquées dans les conflits à titre personnel. Par contraste, les hommes se protègeraient davantage en demeurant à distance : "(..) le rôle du chef d'établissement, à un moment donné, est un rôle de prise de décisions. Ces décisions peuvent être, certaines fois, contestées mais le chef d'établissement se voit contraint de les prendre. Et, il faut savoir les imposer en ayant un peu de poigne. Et je pense que cela amène obligatoirement à des conflits et je ne sais pas si toutes les femmes acceptent de rentrer dans ces conflits. Pour en avoir discuté avec pas mal de collègues, c'est que..., ce n'est pas ma personne qui est impliquée dans le conflit. Je suis le personnel de direction et, à ce titre, je suis obligé de gérer une situation mais le soir, cela ne m'empêche pas de dormir ou, du moins, cela n'empêche pas de dormir le bonhomme qu'il y a derrière le personnel de direction. Alors que beaucoup de collègues féminines, avec qui je discute, ont quand même tendance à avoir une sensibilité un petit peu plus exacerbée, et on touche à la personne en même temps que l'on touche au poste. Moi je ferais cette distinction-là (principal de collège, homme de 54 ans, ancien professeur des écoles). Dans cette perspective, le recours au concept de distance au rôle élaboré par Erving Goffman (2002) pourrait s'avérer pertinent.

\section{Conclusion}

\subsection{Implications}

\subsubsection{Du déni mais chez une minorité masculine}

Les résultats qui viennent d'être présentés permettent tout d'abord de montrer l'hétérogénéité des réponses des personnels (y compris des femmes) sur les questions d'égalité perçue. La teneur des réponses proposées révèle-t-elle des dispositions qui laisseraient supposer une intériorisation des formes traditionnelles de la domination masculine ? Les tendances mises au jour invitent à la plus grande prudence et plaident plutôt pour une réponse négative. Certes, une partie des hommes ne 
répondent pas à la question posée ou soutiennent des positions radicales en rejetant la validité de la statistique relative à l'accès aux postes de chef d'établissement. Mais, il ne s'agit que d'une minorité et, il faut ajouter que les propos les plus virulents ont été tenus par des hommes plutôt âgés qui, manifestement, se montrent moins sensibles que les plus jeunes à la question de l'égalité des sexes. Pour une partie d'entre eux, la question ne se pose pas.

\subsubsection{Interroger l'organisation du travail pour tou.te.s. et en interroger les normes " masculines"}

Comparativement, une majorité des personnes interrogées des deux sexes ont souligné les difficultés d'articuler les différents temps de vie (professionnel, familiaux, personnels). Elles ont également mis l'accent sur la disponibilité et l'engagement que requiert l'exercice de fonction de chef.fe d'établissement. Et, dans ce registre, il semble exister un relatif accord entre les femmes et les hommes qui ont répondu (même si, du point de vue quantitatif, les femmes sont plus nombreuses à évoquer ces aspects). Comme le précise Milewski (2011), ce n'est pas une gestion du temps spécifique aux femmes qui doit être mise en avant mais plutôt une autre organisation du travail (et de son temps) pour tous. Il serait aussi intéressant de mener des études comparatives au niveau européen sur les normes de disponibilité et le temps de travail des personnels de direction à l'instar de la recherche menée auprès d'ingénieur.e.s français.e.s et finlandais.e.s (Bigi, 2019). En Finlande, l'existence d'une norme de disponibilité à la fois limitée et évolutive permet de s'adapter aux contraintes familiales et favorise l'égalité professionnelle (Bigi, 2019).

\subsubsection{Des questions de formation à l'égalité et aux stéréotypes}

Nous pourrions objecter que nos résultats révèlent une évolution allant dans le sens d'une meilleure égalité. Nous pourrions également ajouter que depuis 1984, six conventions interministérielles ont été signées pour améliorer l'égalité entre les sexes au sein du système éducatif français. Des formations ont été organisées pour permettre aux acteurs de terrain - dont les chefs d'établissement de mettre en œuvre les orientations proposées et d'interroger les stéréotypes, la socialisation genrée et les stratégies d'orientation filles/garçons en autre. Ces dispositifs de formation ont pu faire évoluer les conceptions de ces acteurs.

\subsection{Perspectives de recherche}

L'égalité d'accès à certains postes d'encadrement dans l'Éducation nationale progresse sensiblement en France en vingt ans mais, dans le même temps, des inégalités demeurent dans les déroulements de carrière des fonctionnaires concernés. En d'autres termes, les politiques visant une meilleure égalité professionnelle entre les femmes et les hommes commencent à porter leurs fruits, mais elles se révèlent encore relativement impuissantes pour faire évoluer durablement les choix des femmes et des hommes vis-à-vis de l'articulation entre l'implication professionnelle et l'engagement dans les autres sphères de la réalité sociale, notamment au niveau de la parentalité. Il est donc nécessaire de mener des recherches auprès des personnels en croisant simultanément l'ancienneté, l'âge, le nombre d'enfants afin d'avoir des données plus fines permettant d'accéder à des indicateurs d'inégalités qui ont pu se sédimenter progressivement au cours de la carrière (articulation des temps de vie). Il est également fondamental d'interroger les parcours biographiques des personnels mais aussi d'investiguer la qualité de vie au travail perçue par les femmes et les hommes et les solutions qu'ils proposent afin d'améliorer l'égalité professionnelle entre femmes et hommes dans l'Éducation nationale et l'enseignement supérieur. C'est l'objet de notre prochaine recherche : que disent les acteurs-actrices des actions à mener en matière d'égalité. Les champs d'application de ces recherches sont aussi à mettre en lien avec les politiques d'égalité dans la fonction publique et les plans d'actions en faveur de l'égalité femmes/ hommes.

\section{Déclaration de liens d'intérêts}

Les auteurs déclarent ne pas avoir de liens d'intérêts. 


\section{Annexe. L'analyse de contenu : rubriques et catégories}

Question ouverte : "les statistiques nationales révèlent qu'en janvier 2009, 43,8 \% des personnels de direction du second degré sont des femmes. Selon vous, quelles sont les raisons qui expliquent cette représentation inégale des femmes et des hommes dans la fonction? (Développer la réponse) ".

\begin{tabular}{|c|c|}
\hline Rubriques & Catégories \\
\hline \multirow{8}{*}{$\begin{array}{l}\text { R1 - Caractéristiques indivi- } \\
\text { duelles des femmes }(9,2 \%)\end{array}$} & C1 - Elles sont moins ambitieuses que les hommes \\
\hline & C2 - Elles ont moins confiance en elles que les hommes \\
\hline & C3 - Elles ont peur du pouvoir \\
\hline & C4 - Elles s'autocensurent \\
\hline & C5 - Elles aiment moins faire carrière que les hommes \\
\hline & C6 - Elles sous-estiment leurs capacités \\
\hline & $\begin{array}{l}\text { C7 - Elles ont un niveau d'exigence plus élevé que les hommes et } \\
\text { elles se réfrènent davantage avant de s'engager dans la fonction }\end{array}$ \\
\hline & $\begin{array}{l}\text { C8 - Elles craignent de perdre le contact privilégié qu'elles avaient } \\
\text { avec les élèves lorsqu'elles étaient enseignantes }\end{array}$ \\
\hline
\end{tabular}

R2 - Caractéristiques individuelles des hommes $(3,7 \%)$

C9 - Ils sont plus sensibles que les femmes à la reconnaissance qui est associée au poste de direction

C10 - Les hommes sont plus ambitieux que les femmes

C11 - Les hommes sont plus attirés que les femmes par le pouvoir

C12 - Caractéristiques biologiques des hommes

R3 - Difficultés pour concilier l'engagement professionnel, les responsabilités familiales et la vie personnelle $(53,5 \%)$

C13 - Difficultés mentionnées en évoquant son propre cas personnel

C14 - Difficultés mentionnées sans évoquer son propre cas personnel

C15 - Accent mis sur la vie personnelle

R4 - Causes externes (15,6\%) C16 - Caractéristique structurelle de la société française où les inégalités sexuées perdurent

C17 - Poids des attentes sociales vis-à-vis de l'attribution des postes de direction selon le sexe

R5 - Caractéristiques intrinsèques de la fonction de personnel de direction $(52,9 \%)$

C18 - Charge de travail très lourde

C19 - Grande disponibilité

C20 - Obligation de mobilité géographique

C21 - Métier à risques (conflits, violence, etc.)

C22 - Tâches peu gratifiantes

C23 - Isolement professionnel

C24 - Faible rémunération 
R6 - Discussion de la statistique nationale évoquée dans la question $(18,3 \%)$
C25 - Pour minimiser ou relativiser l'ampleur de l'inégalité

C26 - Pour la nier

C27 - Pour suggérer que l'inégalité est encore plus forte car il convient de prendre en considération la répartition par type de poste (chef ou adjoint, catégorie de l'établissement dirigé, etc.)

\section{28 - La question de se pose pas}

C29 - Pour évoquer le vivier de recrutement qui s'avère très féminisé

C30 - Avec une perspective optimiste consistant à souligner l'évolution en cours : la part des femmes augment régulièrement

R7 - Responsabilité de l'institution $(2,8 \%)$

C31 - L'institution facilite implicitement le recrutement des hommes

C32 - Les femmes ne sont pas suffisamment encouragées par l'institution

R8 - Aucune opinion (1,2 \%)

C33 - Ne sais pas

C34 - Sans avis

\section{Références}

Alber, A. (2013). Un plafond de verre plus bas dans la fonction publique ? Travail, genre et sociétés, 30(2), 131. http://dx.doi.org/ $10.3917 /$ tgs.030.0129

Baudelot, C., \& Serre, D. (2006). Les paradoxes d'une satisfaction : ou comment les femmes jugent leur salaire. Travail, genre et sociétés, 15(1), 121-138. http://dx.doi.org/10.3917/tgs.015.0121

Bigi, M. (2019). Le temps de travail des ingénieur.es : genre et normes de disponibilité temporelle en France et en Finlande. Connaissance de l'emploi. Noisy-le-Grand: Centre d'études de l'emploi et du travail.

Cacouault, M., \& Combaz, G. (2011). Les personnels de direction du second degré. Perception du changement, revendications et aspirations des femmes et des hommes chefs d'établissements et adjoints dans les années 2000. Rapport de recherche réalisé pour le centre Henri Aigueperse - UNSA éducation.

Collet, B., \& Keucheyan, R. (2011). Les inégalités liées à l'origine « ethnique ». In M. Forsé \& O. Galland (Eds.), Les Français face aux inégalités et à la justice sociale (pp. 27-39). Armand Colin.

Combaz, G., \& Burgevin, C. (2015). La direction d'école en France : une opportunité pour les femmes d'accéder à un poste à responsabilités ? Travail, genre et société, 34, 131-150.

Coulangeaon, P. (2016). La place des inégalités culturelles dans la genèse des inégalités globales. In O. Galland (Ed.), La France des inégalités. Réalités et perceptions (pp. 289-302). Paris: Presses Universitaires de la Sorbonne.

Duru-Bellat, M., \& Kieffer, A. (2006). Les deux faces - objective/subjective - de la mobilité sociale. Sociologie du Travail, 48(4), 455473. http://dx.doi.org/10.1016/j.soctra.2006.08.001

Forsé, M. (2011). La hiérarchie perçue et souhaitée des rémunérations. In M. Forsé \& O. Galland (Eds.), Les Français face aux inégalités et à la justice sociale (pp. 27-39). Armand Colin.

Forsé, M., \& Parodi, M. (2007). Perception des inégalités économiques et sentiment de justice sociale. Revue de l'OFCE, $102(3), 483$. http://dx.doi.org/10.3917/reof.102.0483

Galland, O., \& Lemel, Y. (2018). Sociologie des inégalités (Collection U) (French Edition). Armand Colin.

Galland, O., Lemel, Y., \& Frénod, A. (2013). La perception des inégalités en France. Revue européenne des sciences sociales, 51-1, 179211. http://dx.doi.org/10.4000/ress.2343

GEMAS (1976). Perception des inégalités sociales et de la justice. Paris: Commissariat général au plan.

Goffman, E. (2002). La " distance au rôle " en salle d'opération. Actes de la recherche en sciences sociales, 143, 80-87.

Gonthier, F. (2011). Hauts et bas revenus : des planchers et des plafonds inégalement consensuels. In M. Forsé \& O. Galland (Eds.), Les Français face aux inégalités et à la justice sociale (pp. 41-49). Armand Colin.

Guichard-Claudic, Y., Kergoat, D., \& Vilbrod, A. (2008). L'inversion du genre. Presses universitaires de Rennes.

Jost, J. T., \& Banaji, M. R. (1994). The role of stereotyping in system-justification and the production of false consciousness. British Journal of Social Psychology, 33(1), 1-27. http://dx.doi.org/10.1111/j.2044-8309.1994.tb01008.x

Latour, E. (2008). Le plafond de verre universitaire : pour en finir avec l'illusion méritocratique et l'autocensure. Mouvements, 5556(3), 53. http://dx.doi.org/10.3917/mouv.055.0053

Laufer, J. (2005). La construction du plafond de verre : le cas des femmes cadres à potentiel. Travail et Emploi, 31-44(102).

Marcel, J.-C., \& Verley, E. (2011). Déclassement et ascension sociale. In M. Forsé \& O. Galland (Eds.), Les Français face aux inégalités et à la justice sociale (pp. 80-81). Armand Colin.

Marry, C. (2014). Inégalités dans le couple et sentiment d'injustice. Les paradoxes de l'égalité contemporaine. In F. Dubet (Ed.), Inégalités et justice sociale (pp. 198-214). Paris: La Découverte. 
Marry, C., Jacquemart, A., Pochic, S., Bereni, L., Le Mancq, F., \& Revillard, A. (2015). Le genre des administrations la fabrication des inégalités de carrière dans la haute fonction publique entre hommes et femmes. Revue française d'administration publique, 153(1), 45. http://dx.doi.org/10.3917/rfap.153.0045

Méda, D., \& Vendramin, P. (2010). Les générations entretiennent-elles un rapport différent au travail ? SociologieS (https://journals. openedition.org/sociologies/3349).

Milewski, F. (2011). L'inégalité entre les femmes et les hommes dans la haute fonction publique : du constat aux moyens d'y remédier. Politiques et management public, 28(2), 157-179. http://dx.doi.org/10.3166/pmp.28.157-179

Richardot, S., Collet, B., \& Frénod, A. (2016). Femmes et hommes : perceptions des inégalités et des discriminations. In O. Galland (Ed.), La France des inégalités. Réalités et perceptions (pp. 83-98). Paris: Presses Universitaires de la Sorbonne.

Sénac-Slawinski, R. (2007). L'ordre sexué. Presses universitaires de France.

Singly, F., \& de Singly, F. (2007). L'injustice ménagère. Armand Colin.

Verniers, C., \& Martinot, D. (2015). L'EJSAG, une Échelle de Justification du système Spécifique Au Genre : validation auprès d'une population d'adolescents et d'adultes. L'Année psychologique, 1-34. http://dx.doi.org/10.4074/s0003503314000049

Weber, M. (1921). Économie et société. Paris, Agora: Édition française collection de poche (1995). 